\title{
Research Progress of Diabetes Mellitus Complicated with Cardiovascular Disease
}

\author{
Yuting Liu* \\ School of Public Health, Zhengzhou University, Zhengzhou 451450, Henan Province, China
}

*Corresponding author: Yuting Liu, 1742274960@qq.com

\begin{abstract}
Diabetes is a chronic disease recognized by the World Health Organization, which is harmful to human life and health. With the improvement of people's quality of life, people's diet structure has changed greatly, and the primary "three high" prevalence rate has risen sharply. The main reason for the impaired life expectancy of a large number of diabetic patients is the threat of a series of cardiovascular complications. According to statistics, the risk of cardiovascular disease in diabetic patients are 2-4 times higher than that in non-diabetic patients. More than $80 \%$ of patients with type 2 diabetes die of cardiovascular disease every year, and $75 \%$ of them have ischemic heart disease ${ }^{[1]}$. All kinds of data show that diabetes mellitus complicated with cardiovascular diseases have become a worldwide public health problem. We must try our best to find a cure mechanism and give patients a healthy life. In order to effectively curb the threat of diabetes complicated with cardiovascular disease patients, we should strengthen the research in this field. Through the analysis of the risk factors of diabetic cardiovascular disease, we can let the majority of medical workers understand the superficial and potential risk factors, to formulate scientific and reasonable treatment plan, for further improving the quality of life of patients. In the following, the author first introduces the basic situation of diabetes and cardiovascular complications, then introduces the main risk factors of diabetic cardiovascular disease one by one, hoping that this study can help to improve the cure rate of diabetes complicated with cardiovascular disease.
\end{abstract}

Keywords: Diabetes; Cardiovascular disease; Risk factors; Occurrence mechanism; Risk assessment

Publication date: July 2021; Online publication: July 31, 2021

\section{Introduction}

According to the statistics of the World Health Organization (WHO), the number of adults with diabetes in the world has reached 463 million by 2019, and it is expected that this number will increase significantly in the future, estimated to reach 700 million by $2045^{[1]}$. According to the data of "Research Report on supply and demand situation and competitive strategy of Chinese diabetes drug industry market from 2020 to 2026," it is shown that, the number of diabetes patients in China will reach 122 million in 2019, ranking the first in the world. At present, China has gradually entered the era of aging, and the middle-aged and elderly population are growing rapidly. Considering the growth trend of elderly patients with type II diabetes, it is estimated that by 2028, there will be more than 149 million cases of diabetes in China, which seems to have become a disaster area ${ }^{[2]}$. Diabetes is a common complication of cardiovascular and nervous system caused by long-term hyperglycemia, among which cardiovascular diseases have the highest mortality. Zhao Yuexia et al. ${ }^{[3]}$ mentioned that, to explore the clinical optimal treatment scheme for elderly patients with type II diabetes complicated with cardiovascular disease, through a large number of clinical trials, it is concluded that effective control of blood glucose and blood lipid levels of patients can greatly reduce the risk of cardiovascular disease. In order to achieve stable blood lipid and blood glucose levels of patients, in addition to the necessary standardized drug treatment, it is also necessary to treat the patients. 
The necessary diet guidance and exercise guidance, let the family members of patients participate in the treatment process, to improve the compliance rate of patients after discharge has high clinical application value. In the following, the author summarizes the research progress of diabetes complicated with cardiovascular disease based on his own work experience and referring to the research results of many scholars, to bring good news to diabetes patients in China and abroad.

\section{Overview of diabetes and cardiovascular complications}

Diabetes mellitus is a kind of metabolic disease with hyperglycemia as the main clinical indication, accompanied by insufficient insulin secretion and function, resulting in glucose, lipid and metabolic disorders. At the same time, patients will also show a series of symptoms, such as elevated blood pressure, glucose and lipid metabolism disorders. According to the analysis of cardiovascular disease inducement, the main risk factors of cardiovascular disease include hyperglycemia, hypertension, diabetes mellitus, diabetes mellitus and diabetes mellitus hyperlipidemia makes the incidence of cardiovascular diseases in patients with diabetes significantly higher than that in the general population ${ }^{[4]}$.

Many patients with diabetes mellitus suffer from multiple organ dysfunction due to long-term metabolic abnormalities. Their heart, eyes, kidneys, blood vessels, nerves and other organs show chronic changes, functional deficiencies, and even failure. Macrovascular (heart disease, hypertension and lower extremity vascular disease), microvascular (diabetic retinopathy, diabetic nephropathy) and neuropathy are the main chronic complications of type 2 diabetes. Among them, heart disease and stroke induced by hypertension are the main causes of death in patients with diabetes. Therefore, the clinical data of patients with coronary artery disease can only show the clinical effect of coronary artery bypass grafting. The former WHO recommended class I treatment for diabetes patients with acute myocardial infarction ${ }^{[5]}$. However, the cost of surgery is higher than that of conventional surgery.

\section{Analysis of risk factors of diabetes mellitus complicated with cardiovascular diseases}

\subsection{Age}

A large number of studies have shown that the most important risk factor of type II diabetes complicated with chronic cardiovascular disease is the age of patients. Patients with arteriosclerosis are becoming more ill as they become older, increasing the risk of cardiovascular complications. Therefore, we should pay special attention to cardiovascular disease screening in older diabetic patients. Wang Wenjuan ${ }^{[7]}$ said that through the clinical trial of 214 patients with type II diabetes mellitus complicated with cardiovascular disease, it shows that with the growth of the patient's age, the body functions gradually decline, and the metabolism will slow down. After complicated with cardiovascular disease, the disease continues to worsen, threatening his life and health. At present, there is no mature scheme to cure diabetes in China and abroad, but through the application of various treatment methods, the blood glucose level can be effectively controlled. Some elderly patients with type II diabetes complicated with cardiovascular disease after hospitalization, blood glucose, blood lipid levels have been effectively controlled, clinical symptoms have improved significantly, but after discharge, due to improper diet, irregular medication, leading to repeated aggravation of the disease. Moreover, some patients do not listen to the doctor's advice, privately stop the drug, will control blood sugar, blood lipids, blood pressure, causing the disease to aggravate, threatening their lives and health.

\subsection{Gender}

Clinical research data shows that before menopause, the incidence of acute coronary syndrome in women was lower than that in men due to the protection of endogenous estrogen, but the risk of postmenopausal women has significantly increased ${ }^{[8]}$. 


\subsection{Smoking history}

Carbon monoxide and tar contained in cigarettes can damage human vascular endothelium, which can increase the risk of thrombosis and atherosclerosis. Secondly, people with long smoking age are more likely to have vasospasm, causing angina pectoris and even myocardial infarction ${ }^{[9]}$.

\subsection{Lipid metabolism disorder}

A large number of clinical research results show that many diabetic patients have lipid metabolism disorder, and the clinical indicators are hypertriglyceridemia, low level of high-density lipoprotein cholesterol, and high level of low-density lipoprotein cholesterol, among which the most common type is low-density lipoprotein cholesterol ${ }^{[10-11]}$.

\subsection{Hypertension and hyperglycemia}

Elevated blood pressure and blood glucose levels are important clinical indicators leading to diabetic patients complicated with cardiovascular disease, which are the most important risk factors for diabetes complicated with cardiovascular disease incidence and mortality ${ }^{[12]}$. Firstly, hypertension combined with hyperglycemia can accelerate the speed of atherosclerosis. Secondly, hypertension can accelerate the development of diabetes in patients with impaired glucose tolerance, thereby increasing the risk of cardiovascular disease. Therefore, strict control of blood pressure and blood glucose levels can moderately reduce the possibility of cardiovascular disease in patients with diabetes.

\section{Pathogenesis of diabetic cardiovascular disease}

When patients have a long history of diabetes, their body will accelerate atherosclerosis due to oxidative stress, metabolic disorders and other ways.

\subsection{Dyslipidemia}

Studies have shown that high density lipoprotein cholesterol is the main risk factor of coronary heart disease. The clinical manifestation of diabetic patients is insulin resistance, which is the main cause of dyslipidemia. When insulin is drug-resistant, the anti-lipolysis effect of insulin will be greatly reduced, and the plasma free fatty acids will be increased, thus stimulating the excessive production of hypertriglycerides and the secretion of very low density lipoprotein. On one hand, very low density lipoprotein can be transformed into low density lipoprotein cholesterol, causing atherosclerosis. On the other hand, very low density lipoprotein (VLDL) can dissociate apolipoprotein A-1 from high density lipoprotein cholesterol (HDL-C), thereby HDL-C can be quickly removed, and the protective effect on blood vessels is lost, thus accelerating the formation of atherosclerotic plaque ${ }^{[13]}$.

\subsection{Accumulation of advanced glycation end products}

Advanced glycation end products (AGES) are the most important metabolites of high glucose toxicity in diabetes mellitus. They are important factors involved in the formation and development of diabetic cardiovascular complications. AGES and cell binding receptors promote the production of oxygen free radicals and proinflammatory cytokines, and participate in the formation and development of diabetic cardiovascular complications. Cardiovascular diseases mediated by advanced glycation end products and cell binding receptors mainly include atherosclerosis, heart failure, cardiomyopathy and microangiopathy ${ }^{[14]}$. Contrarily, AGES activates a series of signal transduction pathways, induce cells to produce a large number of reactive oxygen species, trigger oxidative stress and inflammatory cascade, and promote the occurrence and evolution of diabetes and its complications. The endogenous AGES in patients with persistent hyperglycemia in type II diabetes increased significantly, accelerating the speed of coronary 
atherosclerosis $^{[15]}$.

\subsection{Oxidative stress}

Oxidative stress refers to the accumulation of reactive oxygen species (ROS) and reactive nitrogen in the body with the prolongation of the course of disease, resulting in the imbalance of oxidation and antioxidation, thus causing damage to the body tissue. Most diabetic patients are in the state of oxidative stress, and hyperglycemia mediated ROS production is the main factor of diabetic patients complicated with cardiovascular disease. High levels of glucose are metabolized by aldose reductase and sorbitol dehydrogenase to produce high levels of intracellular polyols and fructose ${ }^{[16-17]}$. Evidence shows that the activity of androgen receptor (AR) pathway in patients with hyperglycemia is enhanced, resulting in abnormal relaxation of vascular endothelium, severe damage of endothelial function, easy to form thrombosis, and finally coronary artery stenosis and plaque deposition.

\section{Risk assessment of diabetic cardiovascular complications}

Coronary heart disease is one of the most common cardiovascular complications of diabetes, and it is also the main cause of increased mortality. The results of clinical trials showed that $40 \%$ of patients had coronary heart disease symptoms 5 years after suffering from diabetes, and there was an increasing trend year by year. Therefore, early screening for patients with type II diabetes can effectively reduce the incidence of cardiovascular disease ${ }^{[18]}$. At present, Chinese medical research groups have carried out research on predictive models of type II diabetes, such as Yu Xuejun and Liu Mingzhe, who used different methods to build predictive models of cardiovascular complications of type II diabetes, which played a positive role in promoting early intervention and prognosis nursing of patients.

\section{Conclusion}

In conclusion, there are many risk factors for diabetic patients complicated with cardiovascular diseases, in addition to patients with primary hypertension, hyperlipidemia, hyperglycemia, including many bad living habits, such as smoking, irregular exercise, improper diet and so on. Therefore, we need to take different countermeasures to control it. The incidence rate and mortality rate of cardiovascular complications in type II diabetes are high. Although there is a very mature early risk prediction model abroad, it is not entirely suitable for Chinese people. It is still not necessary to further explore and study, and develop a systematic evaluation system to effectively assess the risk of cardiovascular risk, reduce incidence rate and mortality rate, and effectively improve patient health and quality of life.

\section{Disclosure statement}

The author declares no conflict of interest.

\section{References}

[1] Yang H, Chen W, Pan Y, 2020, Research Progress on the Treatment of Chronic Kidney Disease and Cardiovascular Disease with Diabetes Mellitus. Journal of Clinical Nephrology, (05):28.

[2] Zhao Y, Chen J, 2009, Research Progress of Cardiovascular Disease in Diabetic Patients. Chinese Journal of Practical Medicine, (09):25.

[3] Sun J, Zhai X, Fu Y, 2004, Research Progress on Mechanism of Diabetes Mellitus Complicated with Cardiovascular Disease. Progress in Cardiovascular Disease, (07):25. 
[4] Chen FM, Li K, 2020, Current Situation and Influencing Factors of Cardiovascular Disease in Patients with Diabetic Nephropathy. China Health Engineering, (12):20.

[5] Zhang CR, 2020, Correlation Between Dietary Red Meat Intake and Type II Diabetes Mellitus Complicated with Coronary Heart Disease. South China Preventive Medicine, (12):20.

[6] Chen H, Zhou J, Zeng Z, Peng X, Wang C, 2020, Effect of Nursing Intervention on Cardiovascular Disease in Hemodialysis Patients with Diabetic Nephropathy. China Rural Health, (09):30.

[7] Wang W, 2020, Clinical Observation and Nursing of Elderly Patients with Type 2 Diabetes Mellitus Complicated with Cardiovascular Disease. Renren Health, (07):23.

[8] Zhou H, Zhou H, Dai W, 2019, Correlation between Glycosylated Serum Albumin and Type II Diabetes Mellitus Complicated with Coronary Heart Disease. Experimental and Laboratory Medicine, (06): 15.

[9] Lu D, 2018, Study on the Correlation between Hyperglycemia and Diabetes Mellitus Complicated with Cardiovascular Disease. Diabetes New World, (12):16.

[10] Wang L, 2018, Study on Influencing Factors of Diabetes Mellitus Complicated with Cardiovascular Disease and Nursing Care. Electronic Journal of clinical medical literature, (09):03.

[11] Zhao M, 2018, Prevention and Nursing of Elderly Diabetes Mellitus Complicated with Cardiovascular Disease. Family Medicine. Medical Treatment and Drug Selection, (04):15.

[12] Liu S, 2016, Analysis of Clinical Characteristics of Newly Diagnosed Type II Diabetes Mellitus Complicated with Cardiovascular Disease. Chinese Medicine and Clinical Journal, (11):15.

[13] Gao F, 2018, Tianjin Type 2 Diabetes Research Based on Latent Class Analysis and SF-8 Scale. Tianjin Medical University, (05): 01.

[14] Liu M, 2016, Study on The Correlation between Hyperglycemia and Diabetes Mellitus Complicated with Cardiovascular Disease. Diabetes New World, (03):16.

[15] Cui Y, 2016, Clinical Analysis of Type II Diabetes Mellitus Complicated with Cardiovascular Disease. Chinese Medicine and Clinical, (02):15.

[16] Xue L, 2015, To Explore the Clinical Characteristics and Treatment Effect of Common Complications in Elderly Patients with Type II Diabetes. Diabetes New World, (11):01.

[17] Liu M, 2015, Study on Nursing Education Intervention of Type II Diabetes Mellitus Complicated with Cardiovascular Disease. China Clinical Nursing, (09): 20.

[18] Yu X, 2016, Observation on Community Intervention of Type II Diabetes Mellitus Complicated with Cardiovascular Disease. Diabetes New World, (09):16. 